\title{
La tecnopedagogía como factor para el desarrollo de la calidad académica
}

Technopedagogy as a factor for the development of academic quality

1 Daniel Xavier Fiallo Moncayo

D https://orcid.org/0000-0001-7643-9763

Universidad de Guayaquil, Guayaquil, Ecuador

fiallom@ug.edu.ec

Artículo de Investigación Científica y Tecnológica Enviado: 15/12/2021

Revisado: $28 / 12 / 2021$

Aceptado: 28/01/2022

Publicado:05/02/2022

DOI: https://doi.org/10.33262/ap.v4i1.1.142
Cítese: Fiallo Moncayo, D. X. (2022). La tecnopedagogía como factor para el desarrollo de la calidad académica. AlfaPublicaciones, 4(1.1), 78-93. https://doi.org/10.33262/ap.v4i1.1.142

ALFA PUBLICACIONES, es una Revista Multidisciplinar, Trimestral, que se publicará en soporte electrónico tiene como misión contribuir a la formación de profesionales competentes con visión humanística y crítica que sean capaces de exponer sus resultados investigativos y científicos en la misma medida que se promueva mediante su intervención cambios positivos en la sociedad. https://alfapublicaciones.com

La revista es editada por la Editorial Ciencia Digital (Editorial de prestigio registrada en la Cámara Ecuatoriana de Libro con No de Afiliación 663) www.celibro.org.ec

Digital

Esta revista está protegida bajo una licencia Creative Commons Attribution Non Commercial No Derivatives

4.0 International. Copia de la licencia: http://creativecommons.org/licenses/by-nc-nd/4.0/ 
Palabras claves:

educación,

estrategias

didácticas, tecnopedagogía,

calidad

educativa.

\section{Keywords:}

education, didactic strategies, technopedagogy, educational quality.

\section{Resumen}

El objetivo de esta investigación es analizar la tecnopedagogías como factor para el desarrollo de la calidad educativa. La metodología está basada en un diseño bibliográfico de tipo documental. Los resultaron se basaron en el análisis de las características de la educación virtual, así como también sobre la metodología tecnopedagógica y la calidad educativa. Como conclusión, el dominio de la educación virtual, el compromiso de los docentes en el desarrollo de los contenidos, la responsabilidad de los estudiantes en el desarrollo de su formación y en el control de sus actividades académicas y el deber de los entes correspondientes, tanto gubernamentales como sector educativo, en generar los espacios tecnológicos para el desarrollo de la educación son las variables más importantes de la calidad educativa, por lo que el desarrollo del modelo tecnopedagógico está dentro de estas circunstancias.

\begin{abstract}
The objective of this research is to analyze technopedagogies as a factor for the development of educational quality. The methodology is based on a bibliographic design of the documentary type. The results were based on the analysis of the characteristics of virtual education, as well as on the technopedagogical methodology and educational quality. In conclusion, the domain of virtual education, the commitment of teachers in the development of content, the responsibility of students in the development of their training and in the control of their academic activities and the duty of the corresponding entities, both Governments as an educational sector, in generating technological spaces for the development of education are the most important variables of educational quality, so the development of the technopedagogical model is within these circumstances.
\end{abstract}

\section{Introducción}

A través de los años han existido diversos métodos pedagógicos que han permitido el desarrollo de técnicas de enseñanza. Estos métodos generan los conocimientos necesarios en las personas para aprender alguna ciencia y arte. En tal sentido, la pedagogía tiene un papel clave en el desarrollo de la humanidad, por medio de los procesos educativos. Este proceso se encarga de formar valores y sentimientos en los sujetos vistos como seres 
sociales, por lo que es un proceso desarrollador que busca la formación de las potencialidades funcionales o facultades de los sujetos de la sociedad (GonzálezAgudelo, 1999).

Por tal motivo, la pedagogía es una parte fundamental en el desarrollo y capacitación del ser humano. Esto proviene de su mismo significado. Etimológicamente pedagogía, se desprende del griego paidos que significa niño y gogia que quiere decir llevar o conducir (Mercado, 2008). Por lo cual, la pedagogía es la teoría y disciplina que comprende, busca la explicación y la mejora permanente de la educación y de los hechos educativos, implicada como está en la transformación ética y axiológica de las instituciones formativas y de la realización integral de todas las personas (Vivas, 2012).

Dentro de esta pedagogía se derivan métodos que fueron evolucionando a lo largo del tiempo y que no han sido erradicados a medida que aparece uno nuevo. Esto lo corrobora González-Agudelo (1999), donde el surgir de un modelo nuevo no sólo negaba los errores del modelo anterior, sino también sus logros. Uno de los modelos pedagógico que han tenido gran influencia ha sido el conductivista. El conductismo establece como función de la escuela el transmitir para instruir, ya que imprime en la memoria los saberes y conocimientos específicos, elaborados y terminados por todas las ciencias (Montoya, 2020).

El conductismo permite desarrollar estrategias y conocimientos de una persona a otra con el fin de que pueda aprender un arte, un oficio, un método o una técnica. El conductivismo depende de una persona que tenga el conocimiento, como el docente, para ser transmitido a otra persona, el estudiante, para lograr un objetivo común. El sujeto que va a las instituciones educativas ya no es un alumno, alguien sin luz que repite la cultura universal imitando a su maestro, sino que es un estudiante que procesa información y la aplica a ciertas situaciones (González-Agudelo, 1999).

Sin embargo, a medida del tiempo se ha detallado que el sujeto receptor de la enseñanza también posee información que proviene de su experiencia previa la cual sirve de materia prima para la generación del conocimiento. Este modelo es llamado constructivismo, el cual está basado en el papel activo del alumno en la construcción de significados, la importancia de la interacción social en el aprendizaje y la solución de problemas en contextos auténticos o reales (Vivas, 2012). De igual manera el sujeto de aprendizaje es expuesto a experiencias de aprendizaje por descubrimiento, permitiéndole construir su nuevo y propio aprendizaje mediante una adecuada organización de la información y de la reconfiguración de su red de conocimientos (Montoya, 2020).

A partir de este desarrollo constructivista se desarrollaron otros métodos que permitieron poder generar un conocimiento más efectivos de la cual se basa el empoderamiento. El constructivismo permite que el docente motive al estudiante construya conocimientos de 
acuerdo con sus intereses, lo cual implica dinamismo, participación activa y motivada en las diferentes acciones de las clases (Mercado, 2008). Esto es la base que se utiliza a través de la educación a distancia y que posteriormente derivo a la educación virtual.

El uso de las Tecnologías de la Información y Comunicación (TIC) han permitido que la educación se realice de forma sincrónica y asincrónica; además de que el estudiante sea el principal protagonista en la búsqueda de la información que será transformada en conocimiento, gracias a las nuevas herramientas pedagógicas que utilizan los docentes. Según Vence (2014):

Las TIC se han convertido en una poderosa herramienta didáctica que suscitan la colaboración en los alumnos, centrarse en sus aprendizajes, mejoran la motivación y el interés, promueven la integración y estimulan el desarrollo de ciertas habilidades intelectuales tales como el razonamiento, la resolución de problemas, la creatividad y la capacidad de aprender a aprender. (p. 4)

Del mismo modo, las TIC tienen una forma importante en el desarrollo de las estrategias de enseñanza y como los estudiantes deben aplicar sus métodos de aprendizaje. Han permitido un cambio significativo a las técnicas pedagógicas tradicionales y las que han evolucionado a partir de esta. Las fortalezas de la vinculación entre las TIC y la educación se describen como: preparación adecuada y oportuna de las clases, lecciones estructuradas y claras, con actividades variadas y alta participación de los estudiantes, atención a la diversidad y las necesidades individuales, utilización de recursos didácticos y la frecuencia de comunicación de resultados de evaluación (Lapeyre, 2014).

Asimismo, facilita la recogida de información, la selección y discriminación de esta para posteriormente organizarla de manera lógica y significativa; además, puede ayudar a mejorar la capacidad de liderazgo al rotar los roles en los grupos de trabajo propuestos en clase (Martín, 2017). A todo lo anterior, se destaca las fortalezas que permiten fomentar la nueva educación a través de la virtualidad. La era de la conectividad es una realidad que empezó desde hace una década y será la proyección al futuro.

Para lograr esta evolución educativa deben existir nuevos mecanismos que las permitan. No solo son las estrategias pedagógicas también deben existir las herramientas tangibles como los equipos y áreas tecnológicas. Es decir, se exige que se implementen propuestas educativas acordes a las exigencias de la vida moderna, con las cuales se pueda hacer frente a la necesidad de incorporar dicha tecnología en favor de los procesos de enseñanza y aprendizaje (Saavedra, 2015).

Ante esta situación, empiezan a aparecer las debilidades del sistema de educación virtual la cual corresponde a las políticas educativas de implementación de tecnología adecuada 
para el desarrollo de esta nueva educación. Esto tiene que ver con las condiciones y características propias de los países y sistemas (desigualdad, pobreza, escuelas sin acceso a recursos y servicios básicos, entre otros) y lo que ello supone en inversión y mantención para el adecuado funcionamiento de estos recursos en todo centro educativo (Román et al., 2011).

De la misma forma, existen otras debilidades más allá de las políticas educativas las cuales corresponde al dominio de las herramientas tecnopedagógicas por parte de los docentes. Esto lo corrobora Fernández et al. (2018) el motivo principal es la falta de preparación tecno-pedagógica de los propios profesores. Esta falta de dominio constituye una de las barreras que impiden la integración de las TIC en su práctica diaria en el aula (Suárez et al., 2013).

Según Prendes et al. (2010):

Asimismo, incluiría el manejo de los conceptos y funciones básicas asociadas a las TIC y el uso de ordenadores personales, la utilización de herramientas de productividad (procesador de textos, hoja de cálculo, presentador) para generar diversos tipos de documentos, el manejo de conceptos y, cómo no, la utilización de herramientas propias de Internet, web y recursos de comunicación sincrónicos y asincrónicos, con el fin de acceder y difundir información y establecer comunicaciones remotas. (p.176).

No obstante, las debilidades pueden transformarse si existe un compromiso por parte de los entes correspondientes influyendo en las políticas y mecanismo educativos en las mejoras tecnológicas, así como también existe una responsabilidad por parte de los docentes en poder adquirir esos conocimientos tecnopedagógicos necesarios para una educación virtual de calidad. Estas situaciones están cambiando poco a poco debido a la forma de educar y de aprender en la actualidad y en el futuro,

Por lo tanto, el objetivo de esta investigación es analizar la tecnopedagogías como factor para el desarrollo de la calidad educativa. La metodología esta basada en un diseño bibliográfico de tipo documental.

\section{Metodología}

Para la realización de esta investigación, se basó en la metodología de la investigación La metodología de esta investigación se baso en un desarrollo bibliográfico-documental donde la búsqueda de información fue a través de documentos web como artículos científicos, trabajos de grado, informes técnicos, entre otros. El desarrollo metodológico se estructuró de la siguiente manera: importancia de la pedagogía, la relación de las TIC 
y la educación, la relevancia de las estrategias tecnopedagógicas, las características de los modelos pedagógicos y la relación que tienen con la calidad académica.

\section{Resultados y Discusión}

\section{Características de la educación virtual}

Una de las características más importantes en la educación actual es la vinculación que existe con las TIC, tanto con las tecnologías conocidas y las nuevas que involucran a las redes sociales, blogs, páginas web y plataformas virtuales educativas. la virtualidad permite romper las fronteras geográficas y del conocimiento, generar la motivación propia en la persona para poder generar los conocimientos necesarios y así desarrollar nuevas técnicas, métodos o algún arte. Es el resultado de la revolución del conocimiento; el internet permite acceder a información oportuna y genera un nuevo reto para la educación con el uso de herramientas digitales (Pérez, 2018).

El internet permite el desarrollo de nuevos métodos pedagógicos. Uno de ellos es el conectivismo, el cual se basa en la unión de la información en diferentes puntos o redes con el fin de generar conocimientos que se pueden actualizar de manera rápida y eficiente, con un grado de responsabilidad en el aprendizaje por parte de la persona interesada. Según Sarmiento et al. (2015), el conectivismo se apoya en el concepto de redes para entender el conocimiento, como un patrón de relaciones; el aprendizaje, como la creación de nuevas conexiones y la habilidad de maniobrar alrededor de redes/patrones existentes. Además, Siemens (2004) comenta que las conexiones en este tipo de método se basan en dos tipos:

(1) Una red externa de nodos fiables (personas, redes sociales, blogs, wikis, entre otros) que configurará la red externa de aprendizaje personal con el objetivo de mantenerse actualizado respecto a un ámbito determinado, identificando patrones y tendencias; (2) Una red cognitiva interna (red neuronal) como soporte del proceso de interpretación o creación de conocimiento a nivel individual.

El conectivismo ha permitido cambiar el aprendizaje basado en el sistema just in case del modelo tradicional al sistema justo a tiempo (just intime). Por este motivo, las universidades deben cambiar el enfoque basado en el contenido por un enfoque más centrado en las habilidades de aprendizaje, donde los estudiantes deban aprender de forma más activa e independiente involucrándolos en la experiencia y en las actividades de aprendizaje (Salvat y Fructuoso, 2015).

Asimismo, este modelo conectivista se fundamenta de la educación en línea, la cual se apoya de las plataformas educativas virtuales. En este sentido, los modelos formativos en línea son modelos que comparte una serie de elementos comunes, didácticos y tecnológicos que proporcionan al estudiante un sistema o entorno virtual de enseñanza 
aprendizaje activo que exige autodisciplina y autocontrol (Pérez, 2018). Del mismo modo Robledo \& Taborda (2013), comentan que las plataformas deben permitir integrar herramientas de producción de recursos, comunicación entre los profesores, entre profesores y alumnos en tiempo real y diferido, administración, gestión de cursos y agentes, creación de grupos de trabajo y comunidades de aprendizaje.

De la misma forma, las herramientas que pueden desarrollarse dentro de las plataformas virtuales de educación son producto de las estrategias que apliquen los docentes para el desarrollo de los conocimientos. Estas estrategias pueden desarrollarse a través de portafolios electrónicos. Los E-portafolios están basado en el Modelo de Aceptación de Tecnología (TAM) y en el Modelo de Éxito del Sistema de Información, donde ayudan a aumentar la autonomía del aprendiz en los ambientes de aprendizaje virtuales (Digión et al., 2016).

Igualmente, los e-portafolios pueden aplicarse en una asignatura ubicada en una plataforma de educación virtual. Por lo cual, estas asignaturas o materias en línea son la base para el desarrollo del modelo conectivista. Es así como, según Ochoa (2016), las materias en línea consisten en el diseño, puesta en práctica y evaluación de un curso y que puede definirse como una educación o formación ofrecida a individuos que están geográficamente dispersos, o que interactúan en tiempos diferidos del docente empleando los recursos en telecomunicaciones.

De lo anterior, se puede decir que la materia en línea pertenece al desarrollo de la educación en línea realizada a través de las plataformas virtuales de educación, todo basado en la metodología tecnopedagógica que busca desarrollar estrategias de aprender haciendo.

Según Sarmiento et al. (2015):

La aplicación de la técnica Aprender - Haciendo propiciará vivencias, construcción del conocimiento individual y grupal, práctica, realimentación de los procesos o actividades desarrolladas en función de las ejecuciones erradas, procesamiento cognitivo, compartir la experiencia con los pares, confrontación de reacciones y experiencias que nutran al grupo y la aplicación inmediata de lo aprendido tanto en los entornos virtuales de aprendizaje como en las actividades propias del ejercicio docente. (pp. 26-27)

\section{Metodología tecnopedagógica y la calidad académica}

Una de las estrategias aplicadas por el modelo conectivista es los métodos tecnopedagógicos. En el 2011, surgió la construcción de los objetos de aprendizaje, integrando el conocimiento entre las áreas de Educación, Interacción Humano Computador e Ingeniería de Software, en vista de la importancia de ser un producto de 
software y educativo al mismo tiempo (Silva et al.,2013). Esta integración de las áreas de conocimiento es lo que le da el carácter tecnopedagógico a esta metodología propuesta, con el objetivo de producir de forma ágil, objetivos de aprendizaje, donde estén presentes las características pedagógicas, tecnológicas y de interacción (Bieliukas \& Sprock, 2013).

El modelo tecnopedagógico se fundamenta en los objetos de aprendizaje. Se pueden definir como recursos didácticos e interactivos en formato digital, desarrollados con el propósito de ser reutilizados en diversos contextos educativos, donde respondan a la misma necesidad instruccional, siendo ésta su principal característica, con el objetivo de propiciar el aprendizaje (Bieliukas \& Sprock, 2013). Para la construcción de los objetos de aprendizaje se deben cumplir con los 7 pasos, que se muestran en la figura 1 y se describe a continuación:

- Diseño Instruccional de los Objetivos de Aprendizaje (OA): se describe el contexto, características de la audiencia, necesidad instruccional, justificación, requisitos previos de la audiencia, objetivo general, objetivos específicos, contenidos, características y tipo de OA, actividades de aprendizaje y la evaluación.

- Modelado de las funcionalidades del OA: se deben crear los diagramas bajo el Lenguaje Unificado de Modelado (UML), específicamente los Casos de Uso y los Objetos del Dominio.

- Modelado de la Interfaz del OA: donde se construye el prototipo de interfaz de usuario.

- Selección de la tecnología a emplear: lenguajes de programación, herramientas y programas.

- Codificación e implementación del OA: empleando las tecnologías seleccionadas.

- Estandarización del OA: donde se construyen los metadatos LOM.

- Aplicación de un Instrumento de Calidad: donde se elige un instrumento de evaluación que se utilizará para determinar el grado de calidad de OA, considerando la presencia e influencia de los aspectos pedagógicos, tecnológicos y de interacción humano computador (Silva-Sprock et al., 2013, p. 6-7). 


\section{Figura 1}

Metodología tecnopedagógica para la construcción ágil de objetos de aprendizaje.

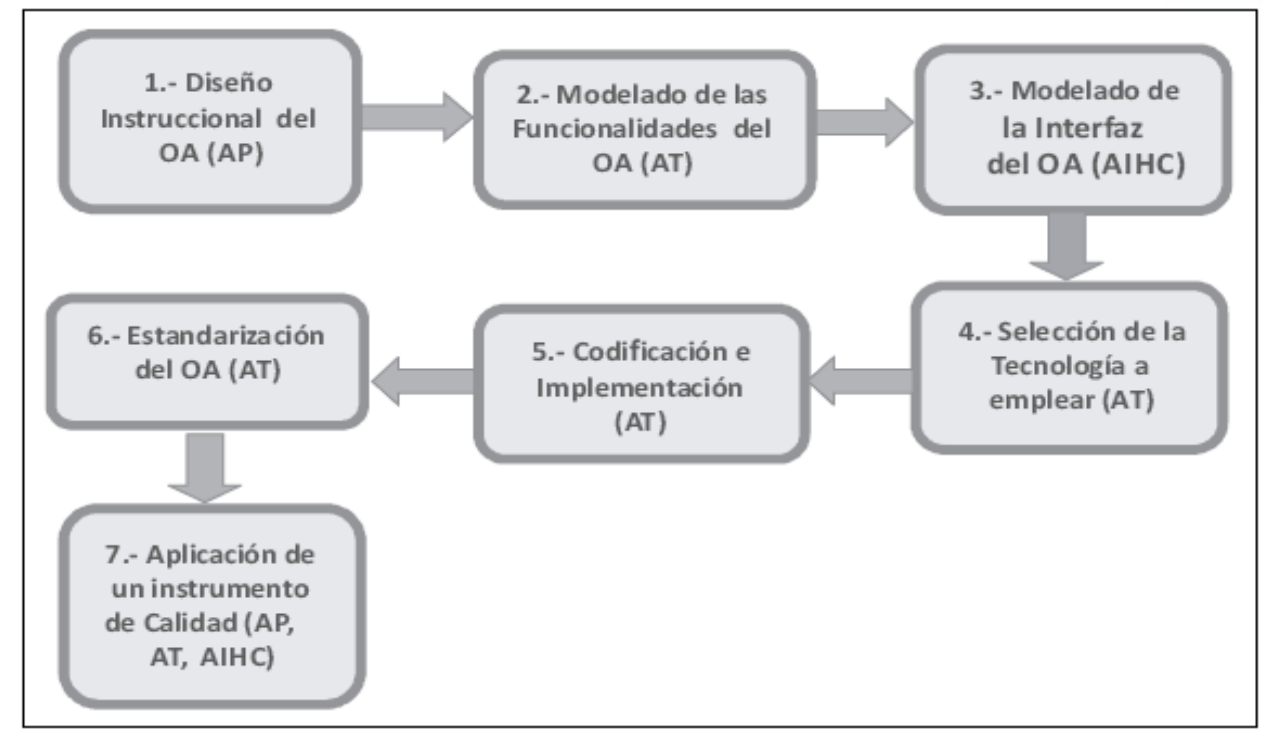

Fuente: Bieliukas \& Sprock (2013)

En consecuencia, el método tecnopedagógico se puede considerar como la construcción de objetos de aprendizaje que determinaran la calidad de la educación ejecutada. Según Bieliukas \& Sprock (2013), considera aspectos pedagógicos, tecnológicos y de interacción humano computador, características que deben estimarse por ser los objetos de aprendizaje un producto de software y educativo al mismo tiempo.

Para poder desarrollar la metodología tecnopedagógica se debe aplicar diversos mecanismos que pretende aumentar la calidad de la educación. Para esto se requiere de una eficiente gestión educativa. La Gestión Educativa es un proceso orientado al fortalecimiento de los Proyectos Educativos, permitiendo mantener la autonomía institucional, en el marco de las políticas estatales, y que enriquece los procesos pedagógicos con el fin de responder a las necesidades educativas (García, 2014).

El control eficiente de la gestión educativa permitirá generar una calidad educativa. La gestión educativa esta entrelaza con las estrategias metodológicas aplicadas por los docentes en los diferentes espacios académicos, sean dentro de un aula de clase o en una materia en línea de una plataforma educativa virtual. En tal sentido, el dominio de las tecnopedagogías pertenece al área que abarca el dominio de la gestión educativa y que debe generar una calidad educativa que permitan una generación de conocimiento productiva y eficiente.

Esto destaca la importancia que tiene dentro de la educación la medición de la calidad de esta. Por lo cual, se debe rescatar como y cuando comenzó a desarrollarse este proceso de 
calidad educativa. Según Quintana-Torres (2018) el término aparece como resultado del informe "A Nation at Risk", de 1983, en el que la educación en Estados Unidos había puesto en peligro la competitividad e integración de la sociedad y que, por lo tanto, era necesario introducir medidas que produjeran cambios.

Según García et al. (2018):

La calidad hace alusión al grado de cumplimiento de objetivos educativos, este modelo fue adoptado del mundo industrial. Se tomó como aspecto positivo la posibilidad de «medir» resultados y como negativo, básicamente, la asociación de la institución educativa con la empresa, lo que no debiera ser así ya que el «producto» aprendizaje es mucho más complejo que cualquier producto empresarial, pues en él existen aspectos y dimensiones difícilmente ponderables, básicamente la relación educativa es una relación humana donde la conducta y circunstancias no son fáciles de estructurar y hasta impredecibles en muchos casos. (p. 210)

En este sentido, la calidad educativa esta entrelazado con los términos de calidad aplicados en múltiples organizaciones, las cuales buscan evaluar el rendimiento y la efectividad de los procedimientos, por lo cual para el ámbito educativo busca evaluar la efectividad de los métodos y estrategias pedagógicas; es decir, evalúa el desempeño del docente a la hora de aplicar las metodologías tecnopedagógicas a razón de su dominio de los objetos de aprendizaje, de la aplicación de herramientas pedagógicas y de la motivación aplicada a los estudiantes. Asimismo, dentro del concepto de calidad de la educación se encuentra la eficiencia social, debido a que el sistema de medición de calidad no toma en cuenta los sistemas actuales de contexto social (Calvo, 2018).

Esto último permite desarrollar una serie de características que involucren todos los aspectos que modifican la educación, pero sobre todo en el progreso y rendimiento académico de los estudiantes.

Según Vidal-Ledo \& Morales-Suárez (2010), estas características son las siguientes:

- Ser accesible.

- Facilitar los recursos personales, organizativos y materiales requeridos.

- Promover cambio e innovación a través de la reflexión compartida sobre la propia práctica docente y el trabajo colaborativo del profesorado.

- Promover la participación activa del alumnado, tanto en el aprendizaje como en la vida de la institución, en un marco de valores donde todos se sientan respetados y valorados como personas.

- Lograr la participación de las familias e insertarse en la comunidad. 
- Estimular y facilitar el desarrollo y el bienestar del profesorado, así como de los demás profesionales del centro. (pp. 253-254)

A estas características sobre calidad educativa se le pueden sumar los principios de calidad los cuales se encuentran en la investigación citada por García et al. (2018), como se mencionan a continuación:

- Hacer bien las cosas desde la primera vez.

- Satisfacer las necesidades del cliente (que para el sector educativo sería el estudiante).

- Buscar soluciones y no justificar errores.

- Ser optimista a ultranza.

- Tener buen trato con los demás.

- Ser oportuno en el cumplimiento de las tareas.

- Ser puntual.

- Colaborar con amabilidad con los compañeros del equipo de trabajo.

- Aprender a reconocer los errores y procurar enmendarlos.

- Ser humilde para aprender y enseñar a otros.

- Ser ordenado y organizado con las herramientas y el equipo de trabajo.

- Ser responsable y generar confianza en los demás.

- Simplificar lo complicado, desburocratizando procesos. (p. 210-211)

En consecuencia, la calidad educativa involucra a las políticas y mecanismos de los entes gubernamentales, pero lo más importante toma con eje central el desarrollo de los docentes en sus dominios tecnopedagógicos. Amavizca (2017), establece que la calidad educativa debe establecer estudios relacionados con la formación inicial y continua, así como evaluaciones que valoren el nivel de dominio de los profesores para los ámbitos de planeación, didáctica, evaluación, y manejo de las herramientas tecnológicas.

\section{Conclusiones}

- Los procesos educativos a lo largo de la historia han ido cambiando a razón de las sociedades y de los ambientes que están alrededor del sistema educativo. La educación siempre ha sido un baluarte, es por ello por lo que siempre las estrategias didácticas van cambiando a razón de como van cambiando las personas y sus necesidades. Se trata de ofrecer una información que será transformada en conocimiento para el desarrollo profesional y personal de los individuos, tal razón es la que determina que la educación es parte fundamental de la humanidad.

- Los modelos pedagógicos han evolucionado a lo largo del tiempo. Han pasado de un modelo tradicional donde el docente es el único que tiene el conocimiento y lo ofrece a los alumnos a través de estrategias pedagógicas básicas. De allí emerge 
lo que se conoce como modelo conductivista, donde el docente aplica sus conocimientos en función de que los estudiantes puedan adquirirlos y así poder desarrollar una habilidad, un oficio o un arte en específico. De estos modelos, el centro de atracción es el profesor, en el cual los estudiantes tienen poco o no tienen conocimientos al respecto.

- Esta situación género que se crearan nuevas expectativas educativas vinculando la experiencia de los estudiantes, lo cual permite el desarrollo del modelo constructivista. Los conocimientos se construyen dentro de los espacios académicos por parte de todos aquellos que hacen vida dentro de la misma. De aquí, nacen más modelos pedagógicos como el cognitivista y el conectivista. Este último tiene gran influencia gracias al auge de las nuevas TIC apoyadas por el avance exponencial del internet.

- El conectivismo utiliza herramientas tecnológicas que permiten el desarrollo del conocimiento de forma sincrónica y asincrónica, en diferentes puntos geográficos. Esta ventaja es la que permite desarrollar métodos didácticos que establezcan estrategias de enseñanza y aprendizaje, donde los docentes y los estudiantes tienen gran responsabilidad de la gestión educativa. Dentro de estos modelos se encuentras la metodología tecnopedagógica, en la cual los entes gubernamentales tienen una gran responsabilidad en las políticas y mecanismos que apoyen en el fortalecimiento de espacios con equipos tecnológicos para el desarrollo de la educación virtual.

- Otra de las aristas que tienen responsabilidad dentro de la educación conectivista son los docentes, por lo que cada profesor debe desarrollar las estrategias didácticas para poder fomentar los conocimientos. Dentro de la vinculación de la educación virtual es necesario que los docentes dominen, no solo la pedagogía sino también las tecnologías que vienen entrelazadas con dichas estrategias. Por tal razón las metodologías tecnopedagógicas deben ser garantizadas en su totalidad. Los estudiantes al pertenecer a la era digital tienen la probabilidad de conocer aún más el desarrollo tecnológico que los profesores, por lo que su dominio en las herramientas y equipos no es un problema en este tipo de educación.

- Los métodos tecnopedagógicos son aquellos que se fundamentan en los objetos de aprendizaje, los mismo que involucran tanto a los equipos tecnológicos como a las estrategias didácticas empleadas por los docentes utilizando dichas herramientas. La puesta a punto de la metodología tecnopedagógica cuenta con el cumplimiento de 7 pasos para el desarrollo de una educación de calidad. Por esta razón, que el desarrollo de las estrategias y dominios de la tecnología por parte de los docentes es uno de los parámetros que abarca la calidad educativa.

- En este sentido, el dominio de la educación virtual, el compromiso de los docentes en el desarrollo de los contenidos, la responsabilidad de los estudiantes en el 
desarrollo de su formación y en el control de sus actividades académicas y el deber de los entes correspondientes, tanto gubernamentales como sector educativo, en generar los espacios tecnológicos para el desarrollo de la educación son las variables más importantes de la calidad educativa, por lo que el desarrollo del modelo tecnopedagógico esta dentro de estas circunstancias.

\section{Referencias Bibliográficas}

Amavizca, K. L. (2017). Estrategia tecno pedagógica para el desarrollo de competencias digitales docentes en profesores universitarios. Cd. Obregón: Tesis para optar al título de Doctora en Sistemas y Ambientes Educativos del instituto tecnológico de sonora.

Bieliukas, Y. C., \& Sprock, A. S. (2013). Una metodología tecnopedagógica para la construcción ágil de objetos de aprendizaje web. Opción, 29(70), 66-85.

Calvo, J. F. (2018). Calidad educativa en la educación superior colombiana: una aproximación teórica. Sophia, 14(2), 4-14.

Digión, L., Figueroa, S. G., \& Noriega, K. (2016). Implementación del portafolio electrónico: una contribución para el aprendizaje en el aula virtual. In XI Congreso de Tecnología en Educación y Educación en Tecnología (TE\&ET 2016).

Fernández-Cruz, F. J., Fernández-Díaz, M. J., \& Rodríguez-Mantilla, J. M. (2018). El proceso de integración y uso pedagógico de las TIC en los centros educativos madrileños. Educación XX1, 21(2). 395-416.

García Colina, F. J., Juárez Hernández, S. C., \& Salgado García, L. (2018). Gestión escolar y calidad educativa. Revista Cubana de Educación Superior, 37(2), 206216.

García-Liscano, R. (2014). Estrategias de gestión educativa desde la perspectiva de la práctica de liderazgo y el fortalecer del buen vivir en la escuela de educación básica "narciso cerda Maldonado", del cantón la maná, provincia de Cotopaxi. Quevedo, Ecuador: Trabajo de grado para optar al título de Magister en Docencia y Currículo de la Universidad Técnica de Babahoyo.

González-Agudelo, E. M. (1999). Un recorrido por los modelos pedagógicos a través de sus didácticas. Reencuentro, 25, 58-68.

Lapeyre, J. (2014). El espacio pedagógico de las TIC. Ponencia presentada al XV Encuentro Internacional Virtual Educa Perú, 9-13. 
Martín, M. M. (2017). Aportaciones pedagógicas de las TIC a los estilos de aprendizaje. Tendencias pedagógicas, (30), 91-104.

Mercado, J. E. (2008). Conceptos básicos en pedagogía. REDHECS: Revista electrónica de Humanidades, Educación y Comunicación Social, 3(4), 36-47.

Montoya, J. (2020). Estudio comparativo de los modelos pedagógicos conductista, cognitivista y constructivista.

Ochoa-Carrasco, I. (2016). Guía práctica los expertos en contenido: desarrollo de materias en línea $(\mathrm{MeL})$. Coordinación de Diseño Instruccional y Formación Tecno-Pedagógica.

Pérez, C. A. (2018). Educación virtual un nuevo desafío. Revista RETO: Revista Especializada En Tecnologías Transversales De La Organización, 6(1), 11-19.

Prendes, M. P., Castañeda, L., \& Gutiérrez, I. (2010). Competencias para el uso de TIC de los futuros maestros. Comunicar, 18(35), 175-182.

Quintana-Torres, Y. E. (2018). Calidad educativa y gestión escolar: una relación dinámica. Educación y educadores, 21(2), 259-281.

Robledo, P. G., \& Taborda, J. A. (2013). Diseño tecnopedagógico de un entorno virtual de aprendizaje para la asignatura de Medicina Legal del Programa de Medicina de la Universidad del Tolima. Revista Perspectivas Educativas, 6., 115-128.

Román, M., Cardemil, C., \& Carrasco, Á. (2011). Enfoque y metodología para evaluar la calidad del proceso pedagógico que incorpora TIC en el aula. RIEE. Revista Iberoamericana de Evaluación Educativa, 4(2), 8-35.

Saavedra, L. E. (2015). Competencias Investigativas en los Docentes Beneficiados por la Estrategia de Formación y Acceso para la Apropiación Pedagógicade las TIC. Tendencias, 16(1), 175-194.

Salvat, B. G., \& Fructuoso, I. N. (2015). Mirando el futuro: Evolución de las tendencias tecnopedagógicas en Educación Superior. Campus virtuales, 2(2), 130-140.

Sarmiento-Alvarado, M., Hernández, M., Villalba, E., \& Zambrano-Steensma, L. (2015). Centro de Asesoría Tecno-Pedagógica UPEL en Línea CATPUL. Proyecto de Grado para optar al Grado de Experto en E-learning de la Universidad Pedagógica Experimental Libertador y la fundación para la actualización tecnológica de Latinoamérica. 
Silva-Sprock, A., Ponce-Gallegos, J. C., \& Hernández-Bieliukas, Y. (2013). Estado del Arte de las Metodologías para el Desarrollo de Objetos de Aprendizaje. Conferencias LACLO, 4(1).

Suárez-Rodríguez, J. M., Almerich, G., Gargallo-López, B., \& Aliaga, F. M. (2013). Las competencias del profesorado en TIC: estructura básica. Educación XX1. 16.1, $39-62$.

Vence, L. (2014). Uso pedagógico de las TIC para el fortalecimiento de estrategias didácticas del programa todos a aprender del ministerio de educación de Colombia. In Congreso Iberoamericano de Ciencia, Tecnología, Innovación y Educación (Vol. 12, p. 13).

Vidal-Ledo, M., \& Morales-Suárez, I. (2010). Calidad educativa. Educación Médica Superior, 24(2), 253-274.

Vivas, J. D. (2012). Modelos pedagógicos en educación a distancia. REDHECS, 12(7), 86-113.

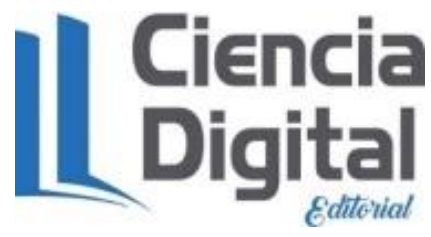


El artículo que se publica es de exclusiva responsabilidad de los autores y no necesariamente reflejan el pensamiento de la Revista Alfa Publicaciones.

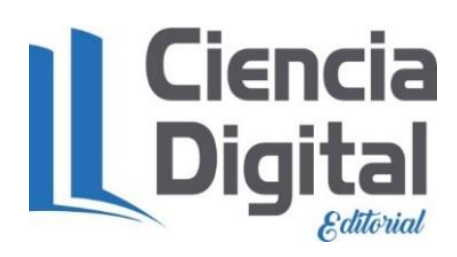

El artículo queda en propiedad de la revista y, por tanto, su publicación parcial y/o total en otro medio tiene que ser autorizado por el director de la Revista Alfa Publicaciones.

$$
\text { latinde }
$$
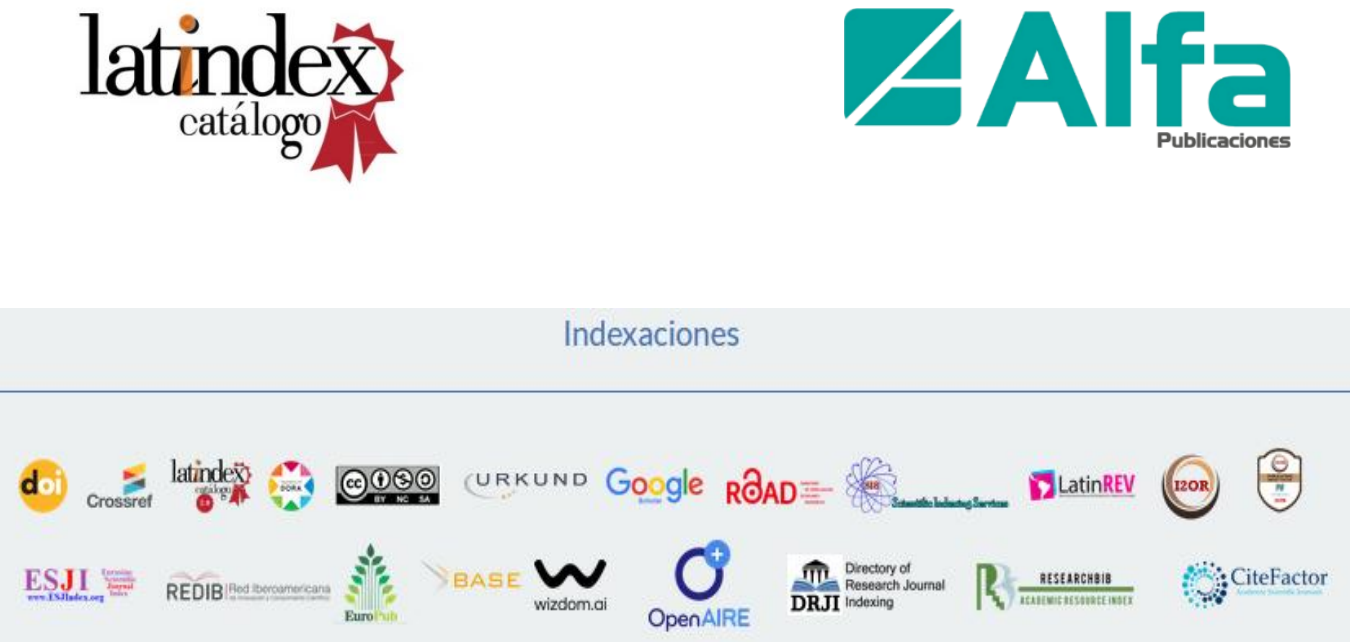